\title{
Compliance in Early-Phase Cancer Clinical Trials Research
}

\author{
RAZELLE KURZRock, a DAVID J. STEWART ${ }^{\text {b }}$ \\ ${ }^{a}$ University of California, San Diego, Moores Cancer Center, San Diego, California, USA; ${ }^{\mathrm{b}}$ Division of Medical Oncology, University of \\ Ottawa, Ottawa, Canada \\ Disclosures of potential conflicts of interest may be found at the end of this article.
}

Key Words. Compliance • Clinical trials

\begin{abstract}
Regulations and ethical principles require that investigators seek consent and that patients participate in experimental studies only under circumstances that minimize the possibility of undue pressure and/or enticements. In recent years, there has been a rapid rise in the monitoring requirements of early-phase trials accompanied by an increasing emphasis on assuring "investigator" compliance with the protocol. It is actually, however, the patient who must comply with the requirements of the study. If there is divergence from the protocol, investigators may be reported to regulatory bodies or agencies. Whereas the investigative
\end{abstract}

community is expected to be vigilant about ensuring that patients participate in studies voluntarily and that their consent is procured without duress, it is also required to guarantee that complex protocols, which entail multiple procedures, be followed exactly by participants who suffer from the complications of advanced cancer. We explore the issue of compliance in a research environment in which investigators are subject to disciplinary action if they fail to ensure that patients adhere precisely to the intense monitoring mandates of a clinical trial. The Oncologist 2013;18: 308-313

Implications for Practice: Ethical principles require that patients participate in experimental studies only under circumstances that minimize the possibility of undue pressure and/or enticements. In recent years, there has been a rapid rise in the monitoring requirements of early-phase cancer clinical trials accompanied by an increasing emphasis on assuring that patients who suffer from advanced cancer nevertheless adhere precisely to the demands of these complex protocols, which entail multiple procedures. We explore the ethical issues of compliance in a research environment in which investigators are subject to disciplinary action and patients may be removed from study if the intense requirements of the protocol are not fully followed.

\section{INTRODUCTION}

Progressively more intensive monitoring is being used in cancer clinical trials research [1,2]. Monitoring of patients in clinical studies via tabulation of adverse events, follow-up of laboratory tests, electrocardiograms (ECGs), scans, and specialized testing (e.g., ophthalmology exams, pharmacokinetic [PK] sampling) generally serves one of two purposes: (a) ensuring patient safety while on an experimental drug and (b) data collection and analysis that are not required for the direct clinical management or safety of the patient but are incorporated to more fully evaluate the drug for research purposes. A subset of the latter group of tests may be necessary to validate the scientific premise of the study.

At the same time that there has been an escalation in study-mandated procedures, especially in early clinical trials, protocol compliance is being increasingly stressed, with studies closely scrutinized for any unplanned variation from the protocol. Many deviations are labeled as violations (and hence, imply seriousness). Study personnel are required to report, explain, and provide corrective action plans to internal review boards (IRBs) for all deviations and violations, and au- ditors are often highly critical of any departure from the protocol. Investigators or staff may be rightfully concerned that deviations or violations could trigger reports to regulatory bodies such as the U.S. Food and Drug Administration (FDA) $[3,4]$.

IRBs are charged by the federal government with assessing the ethics of clinical research trials and the protection of patient safety. An issue that is fundamental to human subject research is whether or not undue influence is involved in a research patient's enrollment and ongoing participation in a clinical research trial. In this regard, one problem that has not been well addressed is the possibility that the level and intensity of testing, and the emphasis on compliance, may produce an environment that promotes pressure tactics for gaining research patients' adherence to the rigorous demands of the study. Such tactics may result in subjects feeling compelled to follow procedures in order to remain in the study, and these may include conscious and subliminal pressures [5]. The fear of being removed from the study sets the context for patient cooperation because they perceive that they will be harmed if they do not. 
We propose that a form of undue pressure is occurring if there is the subtle or overt suggestion that, if patients consent to the trial, they are obliged to deliver on all the data collection mandates and that there can be consequences for noncompliance, including discontinuation of the study drug(s). It is conceivable that patients with a life-threatening or terminal illness who feel strongly about being enrolled in a clinical trial, even for altruistic reasons, and especially for those who are responding to an experimental drug, might be significantly threatened by the suggestion that noncompliance would result in discontinuation of their study participation.

Pressure tactics are more likely to take place when there are repercussions to study investigators and staff if the protocol is not followed precisely as written. This issue is especially relevant in a research environment that includes multiple monitoring requirements and close surveillance of research personnel in the case of any departure from the protocol. Indeed, investigators may be issued warning letters for failure to assure that all assessments required by the protocol are performed, with examples including even research-related testing such as evaluation of circulating tumor cells or PK samples that were obtained at multiple time points but missed on individual days or times [6].

The commentary herein assesses the degree and manner in which the emphasis on compliance in the setting of intense monitoring promotes covert or overt pressure on patients.

Auditor: "The patient missed several pharmacokinetic blood draws. This is a safety issue. He should have been taken off study."

Investigator: "The patient complied with most pharmacokinetic blood draws. This is not a safety issue."

Auditor: "Missing pharmacokinetic draws affects the safety of future patients."

Investigator: "Clinical trial participants are not responsible for the safety of future patients."

Auditor: "The patient signed the consent, which is like a contract; the patient did not fulfill their part."

Investigator: "The consent is not a contract. It informs the patient about the study."

Auditor: "I will report these violations as noncompliance."

\footnotetext{
WHAT ARE THE MONITORING REQUIREMENTS FOR EARLYPhASE TRIALS?

With more targeted agents being developed for cancer therapy, a strategy is evolving to find less toxic or more effective drugs and doses. Perhaps in part as a result of the increasing desire to reduce toxicities, as well as the historic low benefit rate of many drug interventions in oncology, adverse events are closely scrutinized and patients enrolled in clinical trials are intensively monitored. The earliest stage trials, that is, phase I trials, commonly require frequent repetition of a variety of tests or procedures, such as vital sign measurements, physical examinations, ECG monitoring (in order to evaluate
}

QT interval and rhythms), blood draws for PK analyses and for pharmacodynamic impact, non-PK blood draws, eye exams, endocrine follow-up, and other specialized examinations for safety assessment and/or for data collection [1-4]. In phase II and III studies, Roche et. al [2] observed that the work intensity was greater than had been acknowledged previously. Furthermore, study phase and sponsor were identified as significant independent factors that predicted greater workload. Similar to our results [1], Roche and colleagues [2] demonstrated that earlier phase studies and those sponsored by industry were the most intensive.

Overall, however, there are several parties that are culpable for increasingly complex study design, including industry and government sponsors, regulatory agencies, and investigators themselves. It should be noted that investigator-sponsored trials may at times be equally as demanding on patients or even worse than industry-sponsored protocols, especially when such studies are components of peer-reviewed grants, in which case they tend to be heavy in pharmacodynamic assessments to support the hypothesis being tested by other aspects of the grants.

Several authors $[1,2]$ have discussed issues that emerge as a result of the monitoring directive, including an exponential growth in tests, the rigidity of the rules governing the proper follow-up of patients, and duplication of tests on multiple occasions. In a paper published in 2002 [2], it was estimated that one full-time equivalent research staff was required for enrollment of every 10 patients in a moderate-intensity phase III study. However, phase I and phase II studies have more complex requirements than do phase III studies [1,2], and there is reason to believe that study-related procedures have increased, perhaps substantially, in the decade since these data were compiled. Indeed, in reviewing $>10,000$ protocols, Getz et al. [7] determined that, from 1999 to 2005, unique study procedures grew by $6.5 \%$ annually, procedural frequency increased by $8.7 \%$ each year, and the number of eligibility criteria tripled. Further, in an analysis of 49 phase I trials conducted in our program in July 2004 to March 2007, we found a mean of 45 mandated events in the first 28 days of study (mean of 1.6 mandated events per day) [1]. More recently, this number of events was found to be a mean of 105 (median, 104) over the first 4 weeks on study (as determined by review of 15 industrysponsored phase I protocols activated after January 2009; mean of 3.7 mandated events per day) (Kurzrock, unpublished data). (An event was defined as a history and physical, vital sign measurement, blood draw for standard laboratory tests, urine sample collection, blood draw for PK analysis, blood or tissue collection for pharmacodynamic analysis, ECG, adverse event review, imaging study, or specialized test such as eye exam.) Though this testing schedule appears exhaustive, it still underestimates the challenge for both patients and staff, because one blood drawing session (defined as one event) may require multiple tubes for innumerable distinct tests. Additionally, in some protocols, $>40$ mandated events were required in 1 day, usually at the onset of the study.

The question arises as to whether patients suffering from advanced cancer, who are often fatigued, in pain, and emotionally distraught, undergo this degree of testing on a voluntary basis or if acquiescence to protocol demands is achieved only under some degree of duress. Further, it appears legiti- 
mate to raise the concern that patient autonomy could have been breached on occasion in the face of such an extreme testing burden. Is it conceivable that the higher the degree of compliance under such circumstances, the more likely it is that subtle or overt pressure to comply occurs, and that compliance rates approaching $100 \%$ in this setting are highly unlikely to be attained without significant pressure placed on the patient?

\section{WHAT ARE THE INVESTIGATORS' OBLIGATIONS REGARDING} Adherence to the Protocol?

In the U.S., the terms "protocol deviation" and "protocol violation" are not officially defined by the FDA but are often used interchangeably to mean failing to follow the protocol in any way. Whereas in the past a violation might have implied compromise of patient safety or study integrity, currently many studies blur the distinction between a deviation and violation and, in many clinical trials, any excursion from the protocol document is designated a violation.

Clinical investigators are considered to have specific responsibilities with respect to "changes" in a protocol, with "changes" denoting any procedure that differs from that outlined in the protocol (or in other words, any deviation/violation). For instance, when a U.S. clinical investigator signs the study Form 1572, the investigator promises to "conduct the study in accordance with the relevant, current protocol" and commits that he or she "will only make changes in a protocol after notifying the sponsor, except when necessary to protect the safety, the rights, or welfare of subjects" [4]. The clinical investigator must also promptly report to the IRB all "changes in the research activity." Additionally, it is stated that the investigator is prohibited from "making any changes in the research without IRB approval, except where necessary to eliminate apparent immediate hazards to human subjects" [4].

\section{WhAT ARE THE CONSEQUENCES TO INVESTIGATORS AND}

\section{Study Staff as a Result of Deviation From}

\section{THE PROTOCOL?}

In the U.S., when there is divergence from the protocol, the FDA expects that the sponsor prospectively authorizes the exception, and that this authorization is documented in the study records. The proposed exception may also be reported to the IRB for approval. As mentioned above, many protocols classify any deviation as a violation and/or do not clearly delineate between these terms. Deviations/violations require an explanation and a corrective action plan if they are to be approved retrospectively. If protocol deviations/violations become serious, a designation that is not well defined, and investigator "compliance" cannot be obtained, then the sponsor reports the investigator and problem to the FDA.

As a result, investigators, who often also serve as the patient's physician, may nonetheless have little say in the patient's management if there is a need to diverge from the protocol. Furthermore, they may be concerned about the potential threat of being reported to regulatory bodies such as the FDA if there is disagreement and/or noncompliance with the sponsor, and certainly with the IRB. Finally, because of the complexity of clinical trials, and because patients are not always willing or able to comply with all protocol mandates, there are almost inevitably deviations/violations. Indeed, unless subtle or overt pressure is applied to the patient, it seems improbable that many clinical trial participants would submit to all and, in many cases, even a large portion of the demands of these complicated studies. If the definition of undue pressure includes subliminal pressure to choose or act, it is conceivable that many or the majority of patients who fully or near-fully comply with the protocol do so under duress. The situation is exacerbated by the fact that investigators and study staff are held directly or indirectly responsible for deviations/violations and hence feel vulnerable to the charge of noncompliance. In addition, regulatory bodies issue frequent warning letters to investigators. Some of the warning letters are openly accessible on the Internet [6], and these letters undoubtedly have a chilling effect on the investigative community. Warning letters may include citations for not conforming with the protocol when even a small subset of research correlatives performed repeatedly throughout the study are omitted [6]. It is our experience that investigators and their staff are prone to interpreting these warnings as reason to ensure, by whatever means necessary, that patients comply with every protocol requirement.

Beyond the effect on the patient, the question arises as to whether or not such an environment, potentially perceived as threatening by the investigator, can subtly or overtly influence his or her attribution of adverse events or his or her querying the data produced in a publication or elsewhere, especially in the face of disagreement with the sponsor or regulatory bodies.

\section{How Are Patient Care Decisions Made in the}

\section{Context of a Clinical Research TRIAL?}

During the course of a clinical trial, treatment and monitoring requirements are governed by the protocol. This eliminates the need for many routine, day-to-day decisions about the patient's care. However, patients and protocols are complex and, frequently, a medical, logistical, or personal issue arises that may necessitate deviation from the protocol. At such a time, who decides what is the best course of action?

As an example, whereas FDA regulations in the U.S. imply that investigators can deviate from the protocol if it is necessary to protect the safety of the participants, in practice, when such a deviation can occur is unclear, and hence most investigators are encouraged to obtain sponsor permission before any change in protocol is authorized for a patient. Because most sponsors have millions of dollars invested in their product, by definition, any decision they render regarding patient care is in the setting of a highly significant conflict of interest. It is notable that regulations have not addressed this apparent compromise in the potential integrity of decision-making capacity to ensure that the choices made by the sponsor are in the best interest of the patients. The IRB also reviews many of these decisions once the sponsor permits a deviation/violation. However, if the sponsor does not permit the departure from protocol, the request does not generally reach the IRB, because most investigators know that the IRB will, by and large, not approve such requests. Presumably, this type of oversight and/or inflexibility mitigates the possibility of poor decisions by unscrupulous physician investigators. On the other hand, it also delegates much of the authority regarding patient care to individuals who have never seen or spoken to the patient and, in the case of sponsors, to representatives who are entrusted with making good decisions about their 
drug as their primary goal. Increasingly, in order to attenuate the risks entailed in authorizing departures from the protocol, many sponsors permit no deviations/violations, regardless of what the physician may feel is in the best interest of the patient or what the patient may desire. Therefore, patients are left with the choice of withdrawing from the protocol or continuing to participate even if the conditions on protocol are not optimal and could be relatively easily, in some cases, adjusted. If patients perceive the protocol to be beneficial to them, either because their tumor is responding or because it permits them to fight their disease or to contribute to science,

Increasingly, in order to attenuate the risks entailed in authorizing departures from the protocol, many sponsors permit no deviations/violations, regardless of what the physician may feel is in the best interest of the patient or what the patient may desire. Therefore, patients are left with the choice of withdrawing from the protocol or continuing to participate even if the conditions on protocol are not optimal and could be relatively easily, in some cases, adjusted.

removal from study may be seen as an implicit threat of harm. Giving patients such a choice may therefore result in compliance, but such compliance is achieved under duress.

\section{Are Monitoring Requirements in EARly-PhaSe Trials} For Patient Safety or for Research Purposes?

Concerns about early-phase trials have focused on the risk for serious toxicity with minimal benefit from experimental agents. These concerns about participant safety have resulted in an increase in the stringency of regulatory requirements [3, 4]. Even so, in the largest analysis of phase I cancer studies to date, which included almost 12,000 patients, investigators demonstrated that the toxic death rate was $<0.5 \%[8,9]$. This rate is surprisingly low considering that these patients, by definition, are afflicted by terminal, untreatable cancer. Moreover, it may not be possible to decrease the death rate further, because these definitions included deaths that were even possibly drug related $[10,11]$.

In regard to monitoring requirements, the differentiation between tests required for the patient's well being and those needed for tabulation of research results is important, because divergence from the protocol could compromise patient safety in the former case but not in the latter one. However, for tests such as ECGs, which monitor a vital function, the line is often blurred. For instance, when ECGs are performed numerous times in a day or week, claims that these ECGs are for patient safety purposes, rather than for data collection, seem to contradict the tenets of medical practice. Even drugs with serious known cardiotoxicity are rarely monitored by ECGs more than once per month [12], and we are unaware of any credible data documenting the utility of more frequent ECG monitoring for anticancer agents. The contention that the recent marked increase in ECG monitoring required in phase I oncology studies supports research, rather than patient safety purposes, is validated by our analysis of $>8,000$ ECGs performed on $>500$ consecutive patients with cancer who were enrolled in phase I clinical trials at our center [13]. No cardiac morbidity was detected or prevented as a result of this intense monitoring effort. Therefore, although the acquisition of ECG data may be important in order to avoid the approval of an agent with an unacceptable risk-benefit ratio [14], how this ratio is measured in the setting of life-threatening disease should differ from that for less serious conditions, and current evidence does not support the necessity of this exhaustive monitoring for participant safety (and probably not for research purposes either).

Another work-intensive task in early clinical trials revolves around monitoring minor toxicities. Because of the frequency of minor side effects or unrelated "adverse events," their documentation is an important factor responsible for driving current clinical research costs. Further, the difficulty with documenting every fluctuation in minor symptoms, its attribution, and change in grade makes these liable to be perceived as insufficiently documented. Yet, meticulously reporting these often clinically insignificant adverse events lends little to either the safety assessment of the drug or the research integrity of the trial. It might even be argued that a change in paradigm is needed, and that the time to start increasing surveillance for grade 1-2 toxicities is after the agent has been established as highly likely to be useful, so that large amounts of resources are not expended on documenting minor toxicity for agents that end up abandoned because they are ineffective.

\section{Conclusions}

The complexities of clinical trial requirements with investigational drugs highlight a dilemma, that is, how to balance the need for compliance that ensures patient safety and study integrity with the ethical mandate to avoid patient duress. It is clear, for instance, from a wealth of data, that standard-ofcare monitoring of blood counts for myelosuppressive agents immediately before administration of a new cycle of therapy is required to protect a patient's well being. Most physicians would not continue such a drug, regardless of whether it was approved or experimental, in a patient who refused to undergo such monitoring. The ethical reasoning behind such a decision is based on the concept of nonmaleficence, which derives from the maxim of "first, do no harm." Similarly, most physicians would not comfortably continue conventional chemotherapy in patients who would refuse to undergo repeat computed tomography scanning to assess response at some reasonable interval considered standard of care (generally about every 2 months). That is because such response assessment is required in order to balance the potential harm of the therapy against its potential benefit. In clinical trials, it may therefore be reasonable to expect compliance with a few key things, such as documentation of grade 3-5 toxicity, obtaining a tissue sample for crucial biomarkers, and assessing response. On the other hand, many tests that are clearly for research purposes, either directly (such as PK samples) or indirectly because of their frequency, do not meet the criteria for standard of care precisely because they are not necessary for the safety of that patient. These tests, or at least some portion of them, may be important to the study, but mainly for its 
scientific contribution. However, although the scientific integrity of the study is essential, this integrity is generally not compromised if oft-repeated tests are not acquired at all time points. Yet, Our current system fails to differentiate between the few violations that genuinely compromise patient safety or study integrity and the multitude of potential deviations that have no such impact and may, in some cases, be in the patient's best interests. Avoiding such deviations can enormously complicate and compromise patient care in clinical trials, unnecessarily inconvenience patients at a period in their life when time is especially precious to them, and make study management extremely difficult.

In the current state of affairs, problems related to study management are further exacerbated by several common circumstances and assumptions: (a) the performance of "safetyrelated" tests so frequently that they do not add much value (even from a research perspective), (b) the inference that the investigator and/or his or her research staff are negligent if tests are not done, (c) the implication that the patient has been put at risk by the investigator's and/or staff's failure to

Our current system fails to differentiate between the few violations that genuinely compromise patient safety or study integrity and the multitude of potential deviations that have no such impact and may, in some cases, be in the patient's best interests. Avoiding such deviations can enormously complicate and compromise patient care in clinical trials, unnecessarily inconvenience patients at a period in their life when time is especially precious to them, and make study management extremely difficult.

ensure that all tests are performed, (d) the suggestion or warning that the patient may not be permitted to continue the study (for "safety" reasons) if compliance is not maintained, and (e) the threat that the investigator or staff could be reported to regulatory oversight bodies with subsequent disciplinary action if they fail to secure complete patient compliance. Together, these conditions create an environment in which investigators and staff may be obliged to pressure patients to perform numerous research tests under the guise of safety or study integrity. Further, the fact that the distinction between tests done for research or data tabulation purposes and those needed to protect the health of that individual patient is not well recognized by regulatory bodies, and the lack of clarity about which tests are genuinely necessary for study integrity, exacerbates the potential for investigators, their personnel, sponsors, and regulatory bodies to use the alleged safety or study integrity concern as leverage to compel patient compliance with all the requirements of the protocol.
The solutions to this issue may be complex. However, by signing the 1572 Form, the investigator is not only agreeing to conduct the study according to the protocol but also is affirming that he or she has read the protocol and is able to conduct the study as designed. Investigators should be prepared to forego a study if, in all good conscience, it would not be appropriate for the patient population available to the site, or if the study appears to be excessively onerous on the subjects. A quest by investigators and study sites to be a part of the study of a novel compound may contribute to their inability to object to the near-impossible requirements of early-phase studies. However, at times, this quest is driven by a belief, based on preclinical (or early clinical) data, that the drug or drug combination could have significant antitumor activity, making study rejection difficult.

It is well established that denial is common in cancer patients in general [15], as is therapeutic misestimating among phase I study participants in particular [16]. Patients should be free to withdraw consent without fear of any repercussion if they consider their continuing participation in the study to be less than what they desire, or even for no apparent reason. This issue may, however, be complicated by the fact that patients may feel that they are deriving benefit from the study because of a genuine response, or at times because they are more content trying a new drug even if the chance of response is remote, or even because altruism is a goal. Individual review by the IRB of such cases may need to be encouraged in order to ascertain whether or not it is safe and to the patient's benefit to continue in the trial when not all study demands can be met. It may also be important to ensure that the trend to label deviations as violations be modified, and that departures from the protocol that do not compromise patient safety and study integrity clearly be differentiated from those that do.

We conclude that it is time to address whether or not the importance placed on precisely following the protocol in the setting of intense clinical trial monitoring promotes or necessitates pressure tactics inconsistent with the principles protecting human subjects. Furthermore, solutions to the issue of increasingly burdensome studies and prioritization of patient safety and autonomy should be sought by consensus among regulatory bodies, investigators, sponsors, and patients.
Author Contributions
Conception/Design: Razelle Kurzrock, David J. Stewart
Manuscript writing: Razelle Kurzrock, David J. Stewart
Final approval of manuscript: Razelle Kurzrock, David J. Stewart
DisCLOSURES
Razelle Kurzrock: Astrazeneca, Glaxo Smith Kline, Amgen, Roche, Merck (RF); David J. Stewart: Amgen, Align2Action (C/A); Roche, AstraZeneca Taiwan (H); Roche, Pfizer, AstraZeneca (RF).
(C/A) Consulting/advisory relationship; (RF) Research funding; (E) Employment; (H) Honoraria received; (OI) Ownership interests; (IP) Intellectual property rights/inventor/patent holder; (SAB) Scientific advisory board

\section{REFERENCES}

1. Craft BS, Kurzrock R, Lei X et al. The changing face of phase 1 cancer clinical trials: New challenges in study requirements. Cancer 2009;115:1592-1597.

2. Roche K, Paul N, Smuck B et al. Factors affecting workload of cancer clinical trials: Results of a multicenter study of the National Cancer Institute of Can- ada Clinical Trials Group. J Clin Oncol 2002;20:545556.

3. Shalala D. Protecting research subjects-what must be done. N Engl J Med 2000;343:808-810.

4. Department of Health and Human Services. U. S. Food and Drug and Drug Administration. CFR - Code of
Federal Regulations Title 21. CFR 312.53. Available at http://www.accessdata.fda.gov/scripts/cdrh/ cfdocs/cfcfr/CFRSearch.cfm?CFRPart=312\&showFR = 1\&subpartNode=21:5.0.1.1.3.4, accessed December 29, 2012.

5. Orlowski JP, Christensen JA. The potentially co- 
ercive nature of some clinical research trial acronyms. Chest 2002;121:2023-2028.

6. Department of Health and Human Services. U. S. Food and Drug and Drug Administration. Linda D. Bosserman 7/19/11. Warning Letter, July 19, 2011. Available at http://www.fda.gov/ICECl/EnforcementActions/ WarningLetters/ucm264129.htm, accessed December 29, 2012.

7. Getz K. Protocol design trends and their effect on clinical trial performance. Raj Pharma 2008;315316.

8. Horstmann E, McCabe MS, Grochow L et al. Risks and benefits of phase 1 oncology trials, 1991 through 2002. N Engl J Med 2005;352:895-904.
9. Kurzrock R, Benjamin RS. Risks and benefits of phase 1 oncology trials, revisited. N Engl J Med 2005;352:930-932.

10. Stewart DJ, Kurzrock R. Cancer: The road to Amiens. J Clin Oncol 2009;27:328-333.

11. Stewart DJ, Whitney SN, Kurzrock R. Equipoise lost: Ethics, costs, and the regulation of cancer clinical research. J Clin Oncol 2010;28:29252935.

12. Wu AH. Cardiotoxic drugs: Clinical monitoring and decision making. Heart 2008;94:1503-1509.

13. Naing $A$, Veasey-Rodrigues $H$, Hong DS et al. Electrocardiograms (ECGs) in phase I anticancer drug development: The MD Anderson Cancer Center experience with 8518 ECGs. Ann Oncol 2012;23 2960-2963.

14. Morganroth J. Cardiac repolarization and the safety of new drugs defined by electrocardiography. Clin Pharmacol Ther 2007;81:108 113.

15. Vos MS, de Haes JC. Denial in cancer patients, an explorative review. Psychooncology 2007;16: 12-25.

16. Pentz MA, White M, Harvery RD et al. Therapeutic misconception, misestimation and optimism in participants enrolled in phase I trials. Cancer 2012;118:4571-4578

EDITOR'S NOTE: See the accompanying commentary on pages 242-244 of this issue. 